\title{
Fixed Point Results for Weakly $C$-Contraction Mapping in Modular Metric Spaces
}

\author{
Jinwei Zhao*, Qianqian Zhao*, Bo Jin\#, Linan Zhong\# \\ Department of Mathematics, Yanbian University, Yanji, China \\ Email: ${ }^{*} 948197571 @ q q . c o m,{ }^{*}$ zhonglinan2000@126.com
}

How to cite this paper: Zhao, J.W., Zhao, Q.Q., Jin, B. and Zhong, L.N. (2018) Fixed Point Results for Weakly $C$-Contraction Mapping in Modular Metric Spaces. Open Access Library Journal, 5: e4061. https://doi.org/10.4236/oalib.1104061

Received: October 23, 2017

Accepted: January 28, 2018

Published: January 31, 2018

Copyright $\odot 2018$ by authors and Open Access Library Inc.

This work is licensed under the Creative Commons Attribution International License (CC BY 4.0).

http://creativecommons.org/licenses/by/4.0/

\begin{abstract}
In this paper, we introduce the concept of weakly $C$-contraction mapping in modular metric spaces. And we established some fixed point results in $w$-complete spaces. Our results encompass various generalizations of Banach contraction.
\end{abstract}

\section{Subject Areas}

Mathematical Analysis

\section{Keywords}

Modular Metric Spaces Weakly $C$-Contraction Fixed Point Theory

\section{Introduction}

Fixed point theory has absorbed many mathematicians since 1922 with the celebrated Banach contraction principle (see [1]). It is one of the most useful results in nonlinear analysis, functional analysis and topology. Due to its application in mathematics, the Banach contraction principle has been generalized in many directions (see [2] [3] [4]).

Chatteriea in [5] introduced the notion of $C$-contraction which is a generalization of the Banach contraction.

Definition 1.1. [5] A mapping $T: X \rightarrow X$ where $(X, d)$ is a metric space is said to be a $C$-contraction if there exists $\alpha \in\left[0, \frac{1}{2}\right)$ such that for all $x, y \in X$ the following inequality holds:

$$
d(T x, T y) \leq \alpha(d(x, T y), d(y, T x))
$$

${ }^{*}$ Co-first authors.

${ }^{\#}$ Corresponding authors. 
Chatteriea in [5] proved that if $X$ is complete, then every $C$-contraction mapping have a unique fixed point.

The notion of $C$-contraction was generalized to a weak $C$-contraction by Choudhury in [6].

Definition 1.2. [6] Let $(X, d)$ be a metric space and $T: X \rightarrow X$ be a map. Then $T$ is called a weakly $C$-contraction (or a weak $C$-contraction) if there exists $\varphi:[0 \rightarrow \infty)^{2} \rightarrow[0 \rightarrow \infty)$ which is continuous, and $\varphi(x, y)=0$ if and only if $x=y=0$ such that

$$
d(T x, T y) \leq \frac{1}{2}[d(x, T y)+d(y, T x)]-\varphi(d(x, T y), d(y, T x)),
$$

for all $x, y \in X$.

In [6] the author proved that if $X$ is a complete metric space, then every weakly $C$-contraction has a unique fixed point. This fixed point theory was generalized to a complete, partially ordered metric space in [7] and a ordered 2-metric space in [8].

In 2006, Chistyakov introduced the notion of modular metric space in [9]. Recently, there have been many interesting results in the field of existence and uniqueness of fixed point in complete modular metric (see [10] [11]). In this paper, we will establish fixed point theorems for weakly $C$-contraction in modular metric space. The presented results extend some recent results in the literature.

\section{Preliminaries}

Throughout this paper $\mathbb{N}$ will denote the set of natural numbers.

The notion of modular metric space was introduced by Chistyakov in [9] [12] [13], who proved some fixed point results in such kind of spaces.

Let $X$ be a nonempty set. Throughout this paper, for a function $w:(0, \infty) \times X \times X \rightarrow[0, \infty)$, we write

$$
w_{\lambda}(x, y)=w(\lambda, x, y),
$$

for all $\lambda>0$ and $x, y \in X$.

Definition 2.1. [9] Let $X$ be a nonempty set. A function $w:(0, \infty) \times X \times X \rightarrow[0, \infty)$ is said to be a metric modular on $X$ if it satisfies, for all $x, y, z \in X$, the following condition:

1) $w_{\lambda}(x, y)=0$ for all $\lambda>0$ if and only if $x=y$;

2) $w_{\lambda}(x, y)=w_{\lambda}(y, x)$ for all $\lambda>0$;

3) $w_{\lambda+\mu}(x, y) \leq w_{\lambda}(x, z)+w_{\mu}(z, y)$ for all $\lambda, \mu>0$.

If instead of (i) we have only the condition (i')

$$
w_{\lambda}(x, x)=0 \text { for all } \lambda>0, x \in X,
$$

then $w$ is said to be a pseudomodular (metric) on $X$.

An important property of the (metric) pseudomodular on set $X$ is that the mapping $\lambda \mapsto w_{\lambda}(x, y)$ is non increasing for all $x, y \in X$.

Definition 2.2. [9] Let $w$ is a pseudomodular on $X$. Fixed $x_{0} \in X$. The set

$$
X_{w}=X_{w}\left(x_{0}\right)=\left\{x \in X: w_{\lambda}\left(x, x_{0}\right) \rightarrow 0 \text { as } \lambda \rightarrow \infty\right\}
$$


is said to be a modular metric space (around $x_{0}$ ).

Definition 2.3. [14] Let $X_{w}$ be a modular metric space.

1) The sequence $\left\{x_{n}\right\}_{n \in \mathbb{N}}$ in $X_{w}$ is said to be $w$-convergent to $x \in X_{w}$ if and only if $w_{\lambda}\left(x_{n}, x\right) \rightarrow 0$, as $n \rightarrow \infty$ for some $\lambda>0$;

2) The sequence $\left\{x_{n}\right\}_{n \in \mathbb{N}}$ in $X_{w}$ is said to be $w$-Cauchy if $w_{\lambda}\left(x_{m}, x_{n}\right) \rightarrow 0$ as $m, n \rightarrow \infty$ for some $\lambda>0$;

3) A subset $C$ of $X_{w}$ is said to be $w$-complete if any $w$-Cauchy sequence in $C$ is a convergent sequence and its limit is in $C$.

Definition 2.4. [15] Let w be a metric modular on $X$ and $X_{w}$ be a modular metric space induced by $w$. If $X_{w}$ is a w-complete modular metric space and $T: X_{w} \rightarrow X_{w}$ be an arbitrary mapping $T$ is called a contraction if for each $x, y \in X_{w}$ and for all $\lambda>0$ there exists $0 \leq k<1$ such that

$$
w_{\lambda}(T x, T y) \leq k w_{\lambda}(x, y) .
$$

In [15] Chirasak proved that if $X_{w}$ is a $w$-complete modular metric space, then contraction mapping $T$ has a unique fixed point. At the same time, the author proved the following theorem.

Theorem 2.5. [15] Let $w$ be a metric modular on $X, X_{w}$ be a $w$-complete modular metric space induced by $w$ and $T: X_{w} \rightarrow X_{w}$. If

$$
w_{\lambda}(T x, T y) \leq k\left(w_{2 \lambda}(T x, x)+w_{2 \lambda}(T y, y)\right),
$$

for all $x, y \in X_{w}$ and for all $\lambda>0$, where $k \in\left[0, \frac{1}{2}\right)$, then $T$ has a unique fixed point in $X_{w}$. Moreover, for any $x \in X_{w}$, iterative sequence $\left\{T^{n} x\right\}$ converges to the fixed point.

\section{Main Results}

Theorem 3.1. Let $w$ be a metric modular on $X, X_{w}$ be a $w$-complete modular metric space induced by $w$ and $T: X_{w} \rightarrow X_{w}$. If

$$
w_{\lambda}(T x, T y) \leq k\left(w_{2 \lambda}(x, T y)+w_{2 \lambda}(y, T x)\right)
$$

for all $x, y \in X_{w}$ and for all $\lambda>0$, where $k \in\left[0, \frac{1}{2}\right)$, then $T$ has a unique fixed point in $X_{w}$.

Proof. Let $x_{0}$ be an arbitrary point in $X_{w}$ and we write $x_{1}=T x_{0}$, $x_{2}=T x_{1}=T^{2} x_{0}$, and in general, $x_{n}=T x_{n-1}=T^{2} x_{0}$ for all $n \in \mathbb{N}$. If $T x_{n_{0}-1}=T x_{n_{0}}$ for some $n_{0} \in \mathbb{N}$, then $T x_{n_{0}}=x_{n_{0}}$. Thus $x_{n_{0}}$ is a fixed point of $T$. Suppose that $T x_{n-1} \neq T x_{n}$ for all $n \in \mathbb{N}$. For $k \in\left[0, \frac{1}{2}\right)$, we have

$$
\begin{aligned}
w_{\lambda}\left(x_{n+1}, x_{n}\right) & =w_{\lambda}\left(T x_{n}, T x_{n-1}\right) \\
& \leq k\left(w_{2 \lambda}\left(x_{n}, T x_{n-1}\right)+w_{2 \lambda}\left(x_{n-1}, T x_{n}\right)\right) \\
& =k w_{2 \lambda}\left(x_{n-1}, x_{n+1}\right) \\
& \leq k\left(w_{\lambda}\left(x_{n-1}, x_{n}\right)+w_{\lambda}\left(x_{n}, x_{n+1}\right)\right),
\end{aligned}
$$


for all $\lambda>0$ and all $n \in \mathbb{N}$. Hence,

$$
w_{\lambda}\left(x_{n+1}, x_{n}\right) \leq \frac{k}{1-k} w_{\lambda}\left(x_{n}, x_{n-1}\right),
$$

for all $\lambda>0$ and all $n \in \mathbb{N}$. Put $\beta:=\frac{k}{1-k}$, since $k \in\left[0, \frac{1}{2}\right)$, we get $\beta \in[0,1)$ and hence

$$
w_{\lambda}\left(x_{n+1}, x_{n}\right) \leq \beta w_{\lambda}\left(x_{n}, x_{n-1}\right) \leq \beta^{2} w_{\lambda}\left(x_{n-1}, x_{n-2}\right) \leq \cdots \leq \beta^{n} w_{\lambda}\left(x_{1}, x_{0}\right),
$$

for all $\lambda>0$ and each $n \in \mathbb{N}$. Therefore, $\lim _{n \rightarrow \infty} w_{\lambda}\left(x_{n+1}, x_{n}\right)=0$ for all $\lambda>0$. So for each $\lambda>0$, we have for all $\varepsilon>0$ there exists $n_{0} \in \mathbb{N}$ such that $w_{\lambda}\left(x_{n+1}, x_{n}\right)<\varepsilon$ for all $n \in \mathbb{N}$ with $n \geq n_{0}$. Without loss of generality, suppose $m, n \in \mathbb{N}$ and $m>n$. Observe that, for $\frac{\lambda}{m-n}>0$ and for above-mentioned $\varepsilon$, there exists $n_{\lambda /(m-n)} \in \mathbb{N}$ such that

$$
w_{\frac{\lambda}{m-n}}\left(x_{n+1}, x_{n}\right)<\frac{\varepsilon}{m-n},
$$

for all $n \geq n_{\lambda /(m-n)}$. Now we have

$$
\begin{aligned}
w_{\lambda}\left(x_{n}, x_{m}\right) & \leq w_{\frac{\lambda}{m-n}}\left(x_{n}, x_{n+1}\right)+w_{\frac{\lambda}{m-n}}\left(x_{n+1}, x_{n+2}\right)+\cdots+w_{\frac{\lambda}{m-n}}\left(x_{m-1}, x_{m}\right) \\
& <\frac{\varepsilon}{m-n}+\frac{\varepsilon}{m-n}+\cdots+\frac{\varepsilon}{m-n}=\varepsilon,
\end{aligned}
$$

for all $m, n \geq n_{\lambda /(m-n)} \in \mathbb{N}$. This implies $\left\{x_{n}\right\}_{n \in \mathbb{N}}$ is a Cauchy sequence. By the completeness of $X_{w}$, there exists point $x \in X_{w}$, such that $x_{n} \rightarrow x$ as $n \rightarrow \infty$.

By the notion of metric modular $w$ and the contraction of $T$, we get

$$
\begin{aligned}
w_{\lambda}(T x, x) & \leq w_{\frac{\lambda}{2}}\left(T x, T x_{n}\right)+w_{\frac{\lambda}{2}}\left(T x_{n}, x\right) \\
& \leq k\left(w_{\lambda}\left(x, T x_{n}\right)+w_{\lambda}\left(x_{n}, T x\right)\right)+w_{\frac{\lambda}{2}}\left(T x_{n}, x\right) \\
& =k\left(w_{\lambda}\left(x, x_{n+1}\right)+w_{\lambda}\left(x_{n}, T x\right)\right)+w_{\frac{\lambda}{2}}\left(x_{n+1}, x\right),
\end{aligned}
$$

for all $\lambda>0$ and for all $n \in \mathbb{N}$. Taking $n \rightarrow \infty$ in inequality (12), we obtained that

$$
w_{\lambda}(T x, x) \leq k w_{\lambda}(T x, x) .
$$

Since $k \in\left[0, \frac{1}{2}\right)$, we have $T x=x$. Thus, $x$ is a fixed point of $T$. Next, we prove that $x$ is a unique fixed point. Suppose that $z$ be another fixed point of $T$. We note that

$$
\begin{aligned}
w_{\lambda}(x, z) & =w_{\lambda}(T x, T z) \\
& \leq k\left(w_{2 \lambda}(x, T z)+w_{2 \lambda}(z, T x)\right) \\
& \leq k\left(w_{\lambda}(x, z)+w_{\lambda}(z, T z)+w_{\lambda}(z, x)+w_{\lambda}(x, T x)\right) \\
& =2 k w_{\lambda}(x, z),
\end{aligned}
$$

for all $\lambda>0$. Therefore we have 


$$
(1-2 k) w_{\lambda}(x, z) \leq 0 .
$$

Since $1-2 k>0$, we can imply that $x=z$. Therefore, $x$ is a unique fixed point of $T$.

Next, we will introduce the notion of weakly $C$-contraction in modular metric space.

Definition 3.2. Let $\mathrm{w}$ be a metric modular on $X, X_{w}$ be a modular metric space induced by $w$. A mapping $T: X_{w} \rightarrow X_{w}$ is said to be a weak C-contraction in $X_{w}$ if for all $x, y \in X_{w}$ and for all $\lambda>0$, the following inequality holds:

$$
w_{\lambda}(T x, T y) \leq \frac{1}{2}\left(w_{2 \lambda}(x, T y)+w_{2 \lambda}(y, T x)\right)-\varphi\left(w_{\lambda}(x, T y), w_{\lambda}(y, T x)\right),
$$

where $\varphi[0, \infty)^{2} \rightarrow[0, \infty)$ is a continuous mapping such that $\varphi(x, y)=0$ if and only if $x=y$.

Theorem 3.3. Let $w$ be a metric modular on $X, X_{w}$ be a $w$-complete modular metric space induced by $w$. Let $T: X_{w} \rightarrow X_{w}$ be a weak $C$-contraction in $X_{w}$ such that $T$ is continuous and non-decreasing. Then $T$ has a unique fixed point.

Proof. Let $x_{0}$ be an arbitrary point in $X_{w}$ and we write $x_{1}=T x_{0}$, $x_{2}=T x_{1}=T^{2} x_{0}$, and in general, $x_{n}=T x_{n-1}=T^{2} x_{0}$ for all $n \in \mathbb{N}$. If $T x_{n_{0}-1}=T x_{n_{0}}$ for some $n_{0} \in \mathbb{N}$, then $T x_{n_{0}}=x_{n_{0}}$. Thus $x_{n_{0}}$ is a fixed point of $T$. Suppose that $T x_{n-1} \neq T x_{n}$ for all $n \in \mathbb{N}$, we have

$$
\begin{aligned}
& w_{\lambda}\left(x_{n+1}, x_{n}\right)=w_{\lambda}\left(T x_{n}, T x_{n-1}\right) \\
& \leq \frac{1}{2}\left(w_{2 \lambda}\left(x_{n}, T x_{n-1}\right)+w_{2 \lambda}\left(x_{n-1}, T x_{n}\right)\right)-\varphi\left(w_{\lambda}\left(x_{n}, T x_{n-1}\right), w_{\lambda}\left(x_{n-1}, T x_{n}\right)\right) \\
& =\frac{1}{2}\left(w_{2 \lambda}\left(x_{n}, x_{n}\right)+w_{2 \lambda}\left(x_{n-1}, x_{n+1}\right)\right)-\varphi\left(w_{\lambda}\left(x_{n}, x_{n}\right), w_{\lambda}\left(x_{n-1}, x_{n+1}\right)\right) \\
& =\frac{1}{2} w_{2 \lambda}\left(x_{n-1}, x_{n+1}\right)-\varphi\left(0, w_{\lambda}\left(x_{n-1}, x_{n+1}\right)\right) \\
& \leq \frac{1}{2} w_{2 \lambda}\left(x_{n-1}, x_{n+1}\right) \leq \frac{1}{2}\left(w_{\lambda}\left(x_{n-1}, x_{n}\right)+w_{\lambda}\left(x_{n}, x_{n+1}\right)\right),
\end{aligned}
$$

for all $\lambda>0$. The last inequality gives us

$$
w_{\lambda}\left(x_{n}, x_{n+1}\right) \leq w_{\lambda}\left(x_{n-1}, x_{n}\right),
$$

for all $\lambda>0$ and for all $n \in \mathbb{N}$. Thus $\left\{w_{\lambda}\left(x_{n}, x_{n+1}\right)\right\}$ is a decreasing sequence of nonnegative real numbers and hence it is convergent.

For each $\lambda>0$, let

$$
\lim _{n \rightarrow \infty} w_{\lambda}\left(x_{n}, x_{n+1}\right)=r
$$

Letting $n \rightarrow \infty$ in (16) we have

$$
r \leq \lim _{n \rightarrow \infty} \frac{1}{2} w_{\lambda}\left(x_{n-1}, x_{n+1}\right) \leq \frac{1}{2}(r+r)=r .
$$

or, equivalently,

$$
\lim _{n \rightarrow \infty} w_{\lambda}\left(x_{n-1}, x_{n+1}\right)=2 r .
$$


Again, making $n \rightarrow \infty$ in (17), (19) and the continuity of $\varphi$ we have

$$
r \leq \frac{1}{2} 2 r-\varphi(0,2 r)=r-\varphi(0,2 r) \leq r .
$$

And, consequently, $\varphi(0,2 r)=0$. This gives us that $r=0$ by our assumption about $\varphi$.

Thus, for all $\lambda>0$, we have

$$
\lim _{n \rightarrow \infty} w_{\lambda}\left(x_{n}, x_{n+1}\right)=0 .
$$

From the proof of theorem 3.1, we can prove that $\left\{x_{n}\right\}$ is a $w$-Cauchy sequence. By the completeness of $X_{w}$, there exists a point $x \in X_{w}$, such that $x_{n} \rightarrow x$ as $n \rightarrow \infty$.

By the notion of metric modular $w$ and the contraction of $T$, we get

$$
\begin{aligned}
w_{\lambda}(T x, x) \leq & w_{\frac{\lambda}{2}}\left(T x, T x_{n}\right)+w_{\frac{\lambda}{2}}\left(T x_{n}, x\right) \\
\leq & \frac{1}{2}\left(w_{\lambda}\left(x, T x_{n}\right)+w_{\lambda}\left(x_{n}, T x\right)\right) \\
& -\varphi\left(w_{\lambda}\left(x, T x_{n}\right), w_{\lambda}\left(x_{n}, T x\right)\right)+w_{\frac{\lambda}{2}}\left(T x_{n}, x\right) \\
= & \frac{1}{2}\left(w_{\lambda}\left(x, x_{n+1}\right)+w_{\lambda}\left(x_{n}, T x\right)\right) \\
& -\varphi\left(w_{\lambda}\left(x, x_{n+1}\right), w_{\lambda}\left(x_{n}, T x\right)\right)+w_{\frac{\lambda}{2}}\left(x_{n+1}, x\right),
\end{aligned}
$$

for all $\lambda>0$ and for all $n \in \mathbb{N}$. Taking $n \rightarrow \infty$ by (22), we obtained that

$$
w_{\lambda}(T x, x) \leq \frac{1}{2} w_{\lambda}(T x, x)-\varphi\left(0, w_{\lambda}(T x, x)\right) .
$$

This prove that $x=T x$. Thus $x$ is a fixed point of $T$. Next, we prove that $x$ is a unique fixed point. Suppose that $Z$ and $x$ are different fixed points of $T$, then from (15), we have

$$
\begin{aligned}
w_{\lambda}(z, x) & =w_{\lambda}(T z, T x) \\
& \leq \frac{1}{2}\left(w_{2 \lambda}(z, T x)+w_{2 \lambda}(x, T z)\right)-\varphi\left(w_{\lambda}(z, T x), w_{\lambda}(x, T z)\right) \\
& \leq w_{2 \lambda}(x, z)-\varphi\left(w_{\lambda}(z, x), w_{\lambda}(x, z)\right),
\end{aligned}
$$

for all $\lambda>0$ By the property of the $\varphi$, we have $x=z$. Hence $x$ is a unique fixed point of $T$.

Example 3.4 Let $X=\left\{(a, 0) \in R^{2} \mid a \geq 0\right\} \cup\left\{(0, b) \in R^{2} \mid b \geq 0\right\}$. Defined the mapping $w:(0, \infty) \times X \times X \rightarrow[0, \infty)$ by

$$
\begin{gathered}
w_{\lambda}\left(\left(a_{1}, 0\right),\left(a_{2}, 0\right)\right)=\frac{3\left|a_{1}-a_{2}\right|}{\lambda}, \\
w_{\lambda}\left(\left(0, b_{1}\right),\left(0, b_{2}\right)\right)=\frac{\left|b_{1}-b_{2}\right|}{\lambda},
\end{gathered}
$$

and

$$
w_{\lambda}((a, 0),(0, b))=\frac{3 a}{\lambda}+\frac{b}{\lambda}=w_{\lambda}((0, b),(a, 0)) .
$$


We note that if we take $\lambda \rightarrow \infty$, then we see that $X=X_{w}$ and also $T$ and $\varphi$ is define by

$$
\begin{gathered}
T((a, 0))=\left(0, \frac{a}{2}\right), \\
T((0, b))=\left(\frac{b}{24}, 0\right) .
\end{gathered}
$$

and

$$
\varphi(x, y)=\frac{1}{20}(x+y)
$$

We can imply that

$w_{\lambda}(T x, T y) \leq \frac{1}{2}\left(w_{2 \lambda}(x, T y)+w_{2 \lambda}(y, T x)\right)-\varphi\left(w_{\lambda}(x, T y), w_{\lambda}(y, T x)\right)$ for all $x, y \in X$ and all $\lambda>0$.

Indeed, case1. let $x=\left(a_{1}, 0\right), y=\left(a_{2}, 0\right)$, then

$$
\begin{gathered}
w_{\lambda}(T x, T y)=w_{\lambda}\left(T\left(a_{1}, 0\right), T\left(a_{2}, 0\right)\right)=w_{\lambda}\left(\left(0, \frac{a_{1}}{2}\right),\left(0, \frac{a_{2}}{2}\right)\right)=\frac{\left|a_{1}-a_{2}\right|}{2 \lambda}, \\
w_{2 \lambda}(x, T y)=w_{2 \lambda}\left(\left(a_{1}, 0\right), T\left(a_{2}, 0\right)\right)=w_{2 \lambda}\left(\left(a_{1}, 0\right),\left(0, \frac{a_{2}}{2}\right)\right)=\frac{3 a_{1}}{2 \lambda}+\frac{a_{2}}{4 \lambda}, \\
w_{2 \lambda}(y, T x)=w_{2 \lambda}\left(\left(a_{2}, 0\right), T\left(a_{1}, 0\right)\right)=w_{2 \lambda}\left(\left(a_{2}, 0\right),\left(0, \frac{a_{1}}{2}\right)\right)=\frac{3 a_{2}}{2 \lambda}+\frac{a_{1}}{4 \lambda}, \\
w_{\lambda}(T x, T y) \leq \frac{2}{7}\left(w_{2 \lambda}(x, T y)+w_{2 \lambda}(y, T x)\right) .
\end{gathered}
$$

Case 2. let $x=\left(0, b_{1}\right), y=\left(0, b_{2}\right)$, we have

$$
\begin{aligned}
w_{\lambda}(T x, T y)= & w_{\lambda}\left(T\left(0, b_{1}\right), T\left(0, b_{2}\right)\right)=w_{\lambda}\left(\left(\frac{b_{1}}{24}, 0\right),\left(\frac{b_{2}}{24}, 0\right)\right)=\frac{\left|b_{1}-b_{2}\right|}{8 \lambda}, \\
w_{2 \lambda}(x, T y)= & w_{2 \lambda}\left(\left(0, b_{1}\right), T\left(0, b_{2}\right)\right)=w_{2 \lambda}\left(\left(0, b_{1}\right),\left(\frac{b_{2}}{24}, 0\right)\right)=\frac{b_{2}}{16 \lambda}+\frac{b_{1}}{2 \lambda}, \\
& w_{\lambda}(T x, T y) \leq \frac{2}{9}\left(w_{2 \lambda}(x, T y)+w_{2 \lambda}(y, T x)\right) .
\end{aligned}
$$

Case 3. Let $x=(a, 0), y=(0, b)$, then

$$
\begin{gathered}
w_{\lambda}(T x, T y)=w_{\lambda}(T(a, 0), T(0, b))=w_{\lambda}\left(\left(0, \frac{a}{2}\right),\left(\frac{b}{24}, 0\right)\right)=\frac{b}{8 \lambda}+\frac{a}{2 \lambda}, \\
w_{2 \lambda}(x, T y)=w_{2 \lambda}((a, 0), T(0, b))=w_{2 \lambda}\left((a, 0),\left(\frac{b}{24}, 0\right)\right)=\left|\frac{b}{16 \lambda}-\frac{3 a}{2 \lambda}\right|, \\
w_{2 \lambda}(y, T x)=w_{2 \lambda}((0, b), T(a, 0))=w_{2 \lambda}\left((0, b),\left(0 \frac{a}{2}\right)\right)=\left|\frac{b}{2 \lambda}-\frac{a}{4 \lambda}\right|, \\
w_{\lambda}(T x, T y) \leq \frac{2}{5}\left(w_{2 \lambda}(x, T y)+w_{2 \lambda}(y, T x)\right) .
\end{gathered}
$$




$$
\begin{aligned}
\varphi\left(w_{\lambda}(x, T y), w_{\lambda}(y, T x)\right) & =\frac{1}{20}\left(w_{\lambda}\left((x, T y)+w_{\lambda}(y, T x)\right)\right. \\
& =\frac{1}{20}\left[2\left(w_{2 \lambda}(x, T y)+w_{2 \lambda}(y, T x)\right)\right] \\
& =\frac{1}{10}\left(w_{2 \lambda}(x, T y)+w_{2 \lambda}(y, T x)\right) .
\end{aligned}
$$

Hence we have

$$
w_{\lambda}(T x, T y) \leq \frac{2}{5}\left(w_{2 \lambda}(x, T y)+w_{2 \lambda}(y, T x)\right),
$$

for all $\lambda>0$ and $x, y \in X$. And

$$
\begin{aligned}
& \frac{1}{2}\left(w_{2 \lambda}(x, T y)+w_{2 \lambda}(y, T x)\right)-\varphi\left(w_{\lambda}(x, T y), w_{\lambda}(y, T x)\right) \\
& =\frac{1}{2}\left(w_{2 \lambda}(x, T y)+w_{2 \lambda}(y, T x)\right)-\frac{1}{10}\left(w_{2 \lambda}(x, T y)+w_{2 \lambda}(y, T x)\right) \\
& =\frac{2}{5}\left(w_{2 \lambda}(x, T y)+w_{2 \lambda}(y, T x)\right),
\end{aligned}
$$

for all $\lambda>0$ and $x, y \in X$. We can get

$$
w_{\lambda}(T x, T y) \leq \frac{1}{2}\left(w_{2 \lambda}(x, T y)+w_{2 \lambda}(y, T x)\right)-\varphi\left(w_{\lambda}(x, T y), w_{\lambda}(y, T x)\right),
$$

for all $x, y \in X$ and all $\lambda>0$. Thus $T$ is a weakly $C$-contractive mapping. Therefore, $T$ has a unique fixed point that is $(0,0) \in X_{w}$.

On the Euclidean metric $d$ on $X_{w}$, we see that

$$
\begin{aligned}
d\left(T(1,0), T\left(0, \frac{1}{2}\right)\right)> & \frac{1}{2}\left(d\left(T(1,0), T\left(0, \frac{1}{2}\right)\right)+d\left(\left(0, \frac{1}{2}\right), T(1,0)\right)\right) \\
& -\varphi\left(d\left((1,0), T\left(0, \frac{1}{2}\right)\right), d\left(\left(0, \frac{1}{2}\right), T(1,0)\right)\right) .
\end{aligned}
$$

Thus, $T$ is not a weak $C$-contraction on standard metric space.

\section{Conclution}

In this paper, we extend the fixed point results for the weakly $C$-contraction in modular metric space. Moreover, as example, we give a unique fixed point theorem for a mapping satisfying a weak $C$-contractive condition in modular metric space rather than in standard metric space. The main results of this article generalize and unify some recent results given by some authors.

\section{References}

[1] Banach, S. (1992) Sur les opérations dans les ensembles abtraits et leur applications aux équations intégrales. Fundamenta Mathematicae, 3, 133-181.

https://doi.org/10.4064/fm-3-1-133-181

[2] Bakhtin, I.A. (1989) The Contraction Mapping Principle in Almost Metric Space. Functional Analysis, 30, 26-37.

[3] Du, W.S. (2010) A Note on Cone Metric Spaces and Its Equivalence. Nonlinear Analysis. Theory, Methods \& Applications, 72, 2259-2261. 
https://doi.org/10.1016/j.na.2009.10.026

[4] Azam, A., Fssher, B. and Khan, M. (2011) Common Fixed Points Theorems in Complete Valued Metric Spaces. Numerical Functional Analysis and Optimization, 32, 243-253.

[5] Chatterjea, S.K. (1972) Fixed Point Theorems. Comptes Rendus de P Academie bulgare des Sciences, 25, 727-730.

[6] Choudhury, B.S. (2009) Unique Fixed Point Theorem for Weak $C$-Contractive Mapping. Journal of Engineering \& Technology, 5, 6-13.

[7] Harjani, J., López, B. and Sadarangani, K. (2011) Fixed Point Theorems for Weakly $C$-Contractive Mappings in Ordered Metric Spaces. Computers and Mathematics with Applications, 61, 790-769. https://doi.org/10.1016/j.camwa.2010.12.027

[8] Nguyen, V.D. and Vo Tie, L.H. (2013) Fixed Point Theorems for Weakly $C$-Contractions in Ordered 2-Metric Spaces. Fixed Point Theory and Applications, 2013, 161 .

[9] Chistyakov, W. (2006) Metric Modular Spaces and Their Application. Doklady Mathematics, 73, 32-35. https://doi.org/10.1134/S106456240601008X

[10] Afrah, A.A. and Mohamed, A.K. (2013) Fixed Point Results of Pointwise Contractions in Modular Metric Spaces. Fixed Point Theory and Applications, 2013, 163.

[11] Chirasak, M., Wutiphol, S. and Poom, K. (2011) Fixed Point Theorems for Contraction Mappings in Modular Metric Spaces. Fixed Point Theory and Applications, 2011, 93.

[12] Chistyakov, W. (2010) Modular Metric Spaces, I: Basic Concepts. Nonlinear Analysis: Theory, Methods \& Applications, 72, 1-14.

https://doi.org/10.1016/j.na.2009.04.057

[13] Chistyakov, W. (2008) Modular Metric Spaces Generated by F-Modular. Folia Mathematica, 14, 3-25.

[14] Padcharoen, A., Gopal, D., Chaipunya, P. and Kumam, P. (2016) Fixed Point and Periodic Point Results for $\alpha$-Type F-Contractions in Modular Metric Spaces. Fixed Point Theory and Applications, 2016, 39.

https://doi.org/10.1186/s13663-016-0525-4

[15] Chirasak, M., Wutiphol, S. and Poom, K. (2011) Fixed Point Theorems for Contraction Mappings in Modular Metric Spaces. Fixed Point Theory and Applications, 2011, 93. 